\title{
Impact of Minimally Invasive Restorative Techniques on Pregnant Women Oral Health Related Quality of Life: A Randomized Clinical Trial
}

May M. Adham ( $\square$ may.adham00@gmail.com )

Alexandria University Faculty of Dentistry https://orcid.org/0000-0002-5886-646X

Mona K. El Kashlan

Alexandria University Faculty of Dentistry

Wafaa E. Abdelaziz

Alexandria University Faculty of Dentistry

Ahmed S. Rashad

Damanhour University Faculty of Commerce

Research article

Keywords: Papacarie-Duo, Atraumatic Restorative technique, Minimally invasive caries removal, pregnant women, oral health related quality of life, OHIP-14

Posted Date: December 2nd, 2020

DOI: https://doi.org/10.21203/rs.3.rs-117919/v1

License: (9) This work is licensed under a Creative Commons Attribution 4.0 International License.

Read Full License 


\section{Abstract}

Background: Women tend to delay dental treatment due to the misconception about the safety of dental procedures during pregnancy which can negatively affect their quality of life. Minimally invasive restorative techniques offer alternatives for caries treatment and can help improve their oral health related quality of life (OHRQoL) during this stage.

Aim of the study was to compare the effect of chemo-mechanical caries removal method using Papacarie-Duo and Atraumatic Restorative Treatment (ART) on the OHRQoL of pregnant women.

Methods: A randomized controlled clinical trial was conducted in 2019 and included 162 pregnant women visiting public family health centers in Alexandria, Egypt, with mild to moderate dental pain due to caries. Participants were randomly assigned into Papacarie-Duo group $(n=82)$ and ART group $(n=80)$. The outcome variable was percent change in OHRQoL measured by oral health impact profile (OHIP-14). T test/ Mann Whitney $\mathrm{U}$ test were used to compare groups and a multivariable linear regression analysis was conducted to evaluate the factors affecting percent change in OHIP-14 scores after 6 months.

Results: A significant reduction $(P<0.002)$ was noted in OHIP-14 between baseline and 6 months indicating improvement in OHRQoL of pregnant women in Papacarie-Duo and ART groups (16.26\% and $18.91 \%$ respectively) with no significant difference between groups in bivariate analysis $(P=0.120)$. The multiple linear regression model revealed significantly greater reduction in OHIP-14 scores in the Papacarie-Duo than the ART group (regression coefficient $=4.03,95 \%$ confidence interval: $0.652,7.409, P=$ 0.020).

Conclusion: Minimally invasive restorative techniques, such as ART and chemo-mechanical caries removal using Papacarie- Duo can improve the OHRQoL of pregnant women suffering from mild to moderate dental caries.

Trial registration: ID NCT04619264 (https://clinicaltrials.gov/); November 6 2020, retrospective registration. (https://clinicaltrials.gov/ct2/show/NCT04619264?term=NCT04619264\&draw=2\&rank=1)

\section{Background}

Oral health constitutes an essential component of general health and an important indicator for quality of life. Due to the high cost of dental treatment, the global burden of oral diseases is increasing, especially among poor and disadvantaged populations where access to oral care is challenged by the limited resources allocated to higher priority conditions[1]. This is the case in Egypt, where most primary health care facilities, particularly those in remote areas have insufficient dental materials or instruments, with inadequate equipment because of problems related to infrastructure or spare parts[2].

Atraumatic Restorative Treatment (ART) was developed to treat caries outside dental clinics in remote and under-served areas with limited resources[3]. Later on, ART was introduced as part of the Basic 
Package of Oral Care in public oral health services in Tanzania, South Africa and Latin America because of its low cost and acceptance by patients compared to conventional treatment involving anesthetic injections and drilling[4]. Another non-invasive technique is chemo mechanical caries removal using an agent which eliminates infected tissue while maintaining healthy tooth structure without pulp irritation or discomfort. Papacarie is one of the most common chemo-mechanical agents. Papacarie-Duo, the newest version, was developed in 2011 and has improved properties including extended durability, no need for refrigeration and higher viscosity[5].

Pregnancy is associated with hormonal, psychological and physiological changes, in addition to alteration in behavioral and dietary habits. Pregnancy may increase the risk of dental caries initiation or progression due to change in salivary composition, increase in the acidity of saliva due to episodes of gastric reflux or neglect of oral care[6,7]. Pregnant women sometimes tend to delay dental treatment due to misconceptions about its safety during pregnancy and the adverse effect that may be caused by local anesthesia to the fetus[8]. The resulting dental pain due to the delayed treatment may negatively affects pregnant women' quality of life and causes stress to pregnant women[9]. This may be evident in studies comparing the perception of well-being among pregnant and non-pregnant women which showed that it was lower among pregnant women[10,11]. Moreover, studies evaluating the impact of health-related quality of life on pregnancy outcomes, showed that oral diseases were significant factors affecting social and mental well-being[12,13].

Oral health related quality of life $(\mathrm{OHRQOL})$ is defined as "a multidimensional construct that reflects people's comfort when eating, sleeping, and engaging in social interaction; their self-esteem; and their satisfaction with respect to their oral health"[14]. Many instruments have been developed to measure OHRQoL with different items, format of questions and responses[15,16]. Oral Health Impact Profile (OHIP14 ) is one of the most used instruments. It measures the impact of oral diseases on social life and wellbeing[17]. Musskopf et al[11] investigated the impact of receiving periodontal treatment on the change of OHRQoL among pregnant women and reported significant reduction in OHIP-14 scores following treatment. Retori et al[18] assessed the association between oral hygiene habits and OHRQoL among 100 pregnant women and showed that tooth brushing $>2$ times a day was a protective factor against negative impact on OHRQoL.

Providing dental care to pregnant women from modest socio-economic background in low-resource settings helps ensure their wellbeing and improve their quality of life which reflects on their general health and safety as well as the safety of their newborns. The treatment modalities, thus, must be selected in such a way to ensure the least cost and cause minimum anxiety so that to be suitable to such specific settings and this special target group. Non-invasive restorative techniques can be used to treat pregnant women suffering from dental caries. The aim of the present study was to compare the effect of two minimally invasive caries removal modalities, namely Papacarie-Duo and ART on oral health related quality of life among pregnant women in Alexandria, Egypt. The null hypothesis was that there would be no significant difference between using the two modalities on pregnant women's OHRQoL. 


\section{Methods}

\section{Study design}

This study was conducted as part of a randomized, two parallel-arms, controlled clinical trial assessing the impact of the two treatment modalities on dental pain among pregnant women attending family health units/centers, in Alexandria city, Egypt, for their routine prenatal care from January to October 2019. Ethical approval was first obtained from the Research Ethics Committee, Faculty of Dentistry, Alexandria University (IRB 00010556 - IORG 0008839) and the approval of the director of each family healthcare center was then secured to access the obstetrics and gynecology outpatient clinics. Signed written informed consents were obtained after explaining the aim of the study, risks, benefits and confirming confidentiality of the participants' responses. The trial was registered at clinicaltrial.gov NCT04619264. Instructions on proper oral hygiene habits were provided to all participants.

\section{Participants}

Pregnant women were eligible to join the study if they fulfilled the following criteria: being in the first or second trimester, having at least one carious lesion involving dentine with lesion accessible to hand instruments (International Caries Detection and Assessment System score= 5 or 6)[19] and suffering from at least mild dental pain as identified by a score of at least $5 \mathrm{~mm}$ on a Visual Analogue Scale (VAS) [20] 100-mm-long. Pregnant women with acute pulpitis, swelling or fistula as well as uncooperative patients and those who refused to participate were excluded from the study.

Sample size calculation of the original study was based on $5 \%$ alpha error, $20 \%$ beta error and percentage reporting no pain after chemo-mechanical caries removal $=68 \%$ and $35 \%$ after ART $=35 \%[21]$. The number of participants was calculated to be 160 .

\section{Randomization}

Participants were then randomly allocated to either test or control groups using a computer-generated list[22] in a ratio 1:1. The allocation sequence was concealed from the primary researcher in sequentially numbered, opaque, sealed envelopes. Examination, treatment and outcome assessment were performed by the same researcher who had previously undergone training and calibration for restoration evaluation criteria, (Kappa statistic of intra-examiner agreement=0.91). Blinding of participants was not possible due to the difference between the two techniques.

\section{Interventions}

\section{Papacarie-Duo group[23]}

First, tooth was cleaned with a wet cotton pellet. The gel was inserted using disposable syringe tip into the cavity and its color changed from clear to cloudy indicating the presence of infected tissues. After 40 seconds, the softened carious tissue was removed using excavator by gentle pressure. The remaining gel 
was removed with a cotton pellet. The procedure was repeated until there was no change in the gel color indicating that there was no more infected tissue.

\section{ART group[24]}

The tooth was cleaned with a wet cotton pellet to remove debris and plaque and then isolated with cotton rolls to promote a dry environment. Caries was removed using a sharp excavator (Darby-Perry \#220/221, \#17 DE, Hu-Friedy, Chicago, USA) and the cavity was cleaned using a small wet cotton pellet and dried with a dry cotton pellet.

\section{Cavity restoration[25]}

Cavities in both groups were then filled with high viscosity glass ionomer cement (GIC) in an encapsulated form (Riva Self-Cure, SDI Limited, Bayswater, VIC, Australia). A mechanical mixer was used to mix the capsule for 10 seconds. The capsule was placed into the applicator to apply the GIC into the cavity. A gloved finger was used to apply pressure on the GIC for 1 minute, occlusion was checked and excess material was removed.

\section{Outcomes assessment}

Changes in OHRQoL were assessed by measuring changes between the Oral Health Impact Profile-14 (OHIP-14) scores at baseline and after one and six months[17]. The OHIP-14 includes 14 questions in seven dimensions with two questions for each dimension: functional limitation, physical pain, psychological discomfort, physical disability, psychological disability, social disability and handicap. The present study used the Arabic version of OHIP-14 which was translated by Al Habashneh et al[26] and tested for validity and reliability. Responses to the OHIP-14 items were measured on a 5-point Likert scale ranging from never $=0$; hardly ever $=1$; occasionally $=2$; fairly often $=3$; to very often $=4$. The OHIP-14 score is the sum of the scores of the 14 statements and ranges from 0 to 56 with higher scores indicating higher frequency of negative impact[17].

The clinical evaluation of the restorations was done based on the retention of the GIC in the cavity following the criteria of ART reported by Loe et al[27]. The scoring system was as follows: $0=$ present, without defect; 1 =present, small defects on the margin measuring less than $0.5 \mathrm{~mm}$ in depth, with no need for repair; 2=present, small defects on the margin measuring 0.5 to $1.0 \mathrm{~mm}$ in depth, with need for repair; 3=present, gross defects on the margin measuring $1.0 \mathrm{~mm}$ or more in depth, with need for repair; $4=$ absent, restoration completely lost, need for treatment; $5=a b s e n t$, other treatment had been performed for some reason; $6=$ tooth absent for some reason; $7=$ present, wear on surface less than $0.5 \mathrm{~mm}$, with no need for replacement; 8=present, wear on surface greater than $0.5 \mathrm{~mm}$, with need for replacement; $9=$ diagnosis impossible. Restorations having a score of 0,1 or 7 were considered successful and those receiving a score of 2, 3, 4 or 8 were considered failures. Those receiving scores of 5, 6 or 9 were excluded from the analysis[28]. 
Demographic data (age and education), data about pregnancy related variables (pregnancy stage and order of pregnancy) and other potential confounders like last dental visit, frequency of toothbrushing, perceived state of teeth and gingiva were collected using the Arabic version of the WHO oral health assessment questionnaire for adults with proven validity and reliability[29]. Clinical examination was done to assess the number of decayed teeth based on the WHO criteria[30], the amount of dental plaque and the gingival condition were assessed using Silness and Loe[31] and Loe and Silness[32] criteria.

\section{Statistical analysis}

SPSS (Version 24.0, IBM Corp., Armonk, N.Y., USA) was used for data analysis. Quantitative variables were checked for normality using Shapiro Wilks tests, histograms and QQ plots. Descriptive statistics were displayed as frequencies and percentages for categorical variables and means and standard deviations for quantitative variables. Chi-square and t tests were used to compare baseline characteristics between the two groups (age, education, months of pregnancy, order of pregnancy, brushing frequency, last dental visit, perceived state of teeth and gingiva, number of decayed teeth, gingival index and plaque index). Internal consistency of OHIP-14 items was assessed using Cronbach'a alpha before calculating the domains' and overall scores. An intention-to-treat analysis was performed where patients who were lost to follow up were given the worst score on OHIP-14 and restorations were considered as failure.

Percent reduction in OHIP-14 score was calculated by subtracting OHIP-14 scores at baseline from OHIP14 scores after 6 months divided by OHIP-14 scores at baseline and multiplying by 100. T test was used to assess the association between percent reduction in OHIP-14 scores and restoration success.

Differences in OHIP-14 scores at each time point between the two groups were assessed using independent $t$ test. Mann Whitney $\mathrm{U}$ test was used to compare the percent change in each dimension of the OHIP-14 between groups. Repeated measures ANOVA with Bonferroni correction followed by Post Hoc test was used to assess change in the OHIP-14 across time in each group. Significance level was set at $\mathrm{p}<0.05$.

Multivariable linear regression was conducted to evaluate the factors affecting percent reduction in OHIP14 scores after 6 months. The independent variables in the model were selected based on the framework presented in Figure 1 following previous researches reporting a relationship with OHRQoL[33-36]. These independent variables were grouped into; clinical conditions which included gingival index, plaque index and number of decayed teeth, intervention involving treatment with either Papacarie-Duo or ART, pregnancy-related variables such as pregnancy trimester and history of previous births and finally other possible non-pregnancy-related confounders which is concerned with age and education level.

\section{Results}

A total of 162 women fulfilled the inclusion criteria and were randomly allocated to either Papacarie-Duo group ( $n=82)$ or ART group $(n=80)$. The number of participants lost to follow up at each stage is shown in figure 2. Drop-out rate after 6 months was $14.6 \%$ and $17.5 \%$ respectively. 
The mean age for the Papacarie-Duo and ART group were $27.06 \pm 2.82$ and $26.3 \pm 3.18$ respectively, $\mathrm{P}=$ 0.108 . Most participants received secondary education ( $58.5 \%$ and $63.8 \%, P=0.744)$, were in the second trimester $(64.6 \%$ and $73.8 \%, P=0.209)$ and were pregnant in the second baby $(42.7 \%$ and $46.3 \%, P=$ 0.719 , Table 1) with no significance difference between both groups.

\begin{tabular}{|c|c|c|c|}
\hline & Papacarie-Duo & ART & \\
\hline & mean(SD) & mean(SD) & $\mathbf{P}$ \\
\hline \multirow[t]{2}{*}{ Age } & 27.1(2.82) & 26.3(3.18) & 0.108 \\
\hline & $n(\%)$ & $\mathrm{n}(\%)$ & \\
\hline Education & & & 0.744 \\
\hline Primary/secondary education & $60(73.2 \%)$ & $60(75 \%)$ & \\
\hline University education & $22(26.8 \%)$ & $20(25 \%)$ & \\
\hline Stage of pregnancy & & & 0.209 \\
\hline First trimester & $29(35.4 \%)$ & $21(26.3 \%)$ & \\
\hline Second trimester & $53(64.6 \%)$ & $59(73.8 \%)$ & \\
\hline Order of pregnancy & & & 0.719 \\
\hline First child & $23(28 \%)$ & $18(22.5 \%)$ & \\
\hline Second child & $35(42.7 \%)$ & $37(46.3 \%)$ & \\
\hline Third or more & $24(29.3 \%)$ & $25(31.3 \%)$ & \\
\hline
\end{tabular}

There were no statistically significant differences between groups in brushing frequency $(P=0.155)$, time of last dental visit $(P=0.308)$ or perceived state of teeth $(P=0.157)$. Also, there were no significant differences between groups in the number of decayed teeth $(P=0.150)$, and plaque accumulation $(P=$ 0.428). However, women in the Papacarie-Duo group had significantly greater gingival inflammation than those in the ART group (mean=1.54 and 1.43, P=0.025). Restoration success in Papacarie-Duo and ART groups were $82.9 \%$ and $78.8 \%$, respectively $(P=0.499$, Table 2$)$. 


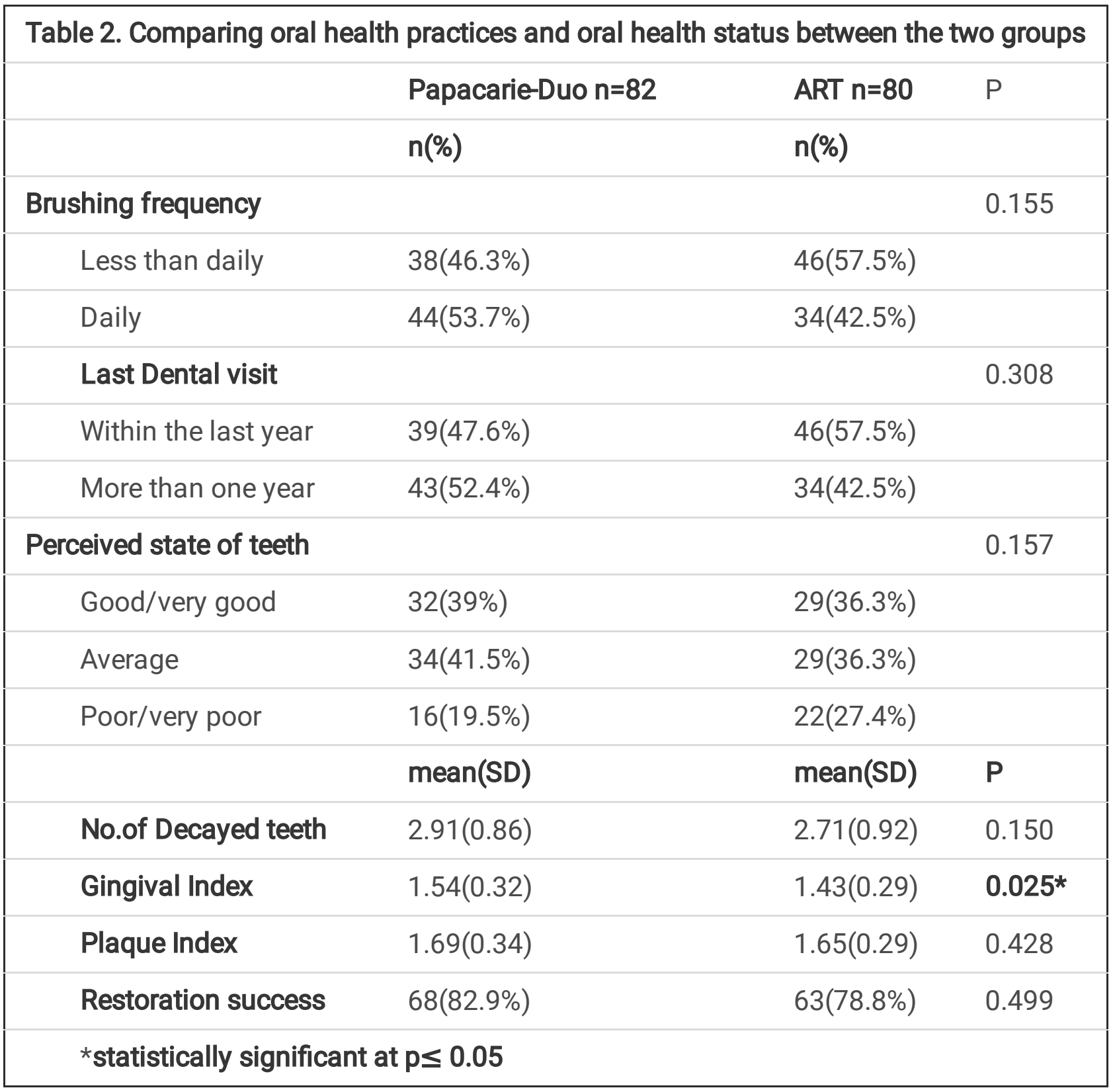

Table 3 presents the scores of the OHIP-14 in the two study groups at different time points. There were no differences between the Papacarie-Duo and ART groups at baseline (19.51 \pm 7.37 and 19.76 $\pm 7.25, P=$ $0.828)$, at one month $(16.95 \pm 6.41$ and $16.65 \pm 6.07, \mathrm{P}=0.74)$ or after six months $(16.18 \pm 6.25$ and $15.73 \pm 5.63, P=0.607)$. In both groups, OHIP-14 score significantly decreased across time $(P<0.001)$ showing significant difference between successive time points in each group, with percent reduction= $16.17 \%$ and $18.91 \%$ respectively after 6 months and no significant difference between both groups ( $P=$ $0.120)$. 


\begin{tabular}{|llll|}
\hline \multicolumn{4}{|l|}{ Table 3. Comparison of OHIP-14 between groups and across time } \\
\hline \\
\hline
\end{tabular}

The Cronbach's alpha of all OHIP-14 items was 0.89 , indicating high internal consistency.

Significantly greater reduction in OHIP-14 score was noted in patients who had successful restorations than patients with failed ones (mean=19.04 and 11.35, $\mathrm{P}=0.004$ ).

Table 4 shows the multivariable linear regression model with the dependent variable being percent reduction in OHIP-14 scores after 6 months. The model including all factors significantly accounted for $11 \%$ of the variation in OHIP-14 reduction among participants (adjusted $R^{2}=0.112, F=3.25, P=0.001$ ). The type of intervention was a significant factor having a significant impact on the reduction in OHIP-14 scores $(P=0.020$, ) where those in the Papacarie-Duo group had 4.03 greater reduction in OHIP-14 scores than those in ART. None of the other pregnancy factors, clinical factors or confounders were significantly associated with OHIP-14 reduction $(P>0.05)$. Plaque index was removed from the model due to collinearity with the gingival index. 


\begin{tabular}{|c|c|c|}
\hline & $\mathrm{B}(95 \% \mathrm{Cl})$ & $P$ value \\
\hline Age & $-0.647(-1.361,0.066)$ & 0.075 \\
\hline \multicolumn{3}{|l|}{ Education level } \\
\hline Less than university & $2.04(-1.701,5.781)$ & 0.283 \\
\hline University or higher & Reference & \\
\hline OHIP-14 at baseline & $-0.128(-0.488,0.231)$ & 0.482 \\
\hline \multicolumn{3}{|l|}{ Pregnancy related variables } \\
\hline \multicolumn{3}{|l|}{ Trimester } \\
\hline First & $1.538(-1.972,5.048)$ & 0.388 \\
\hline Second & Reference & \\
\hline \multicolumn{3}{|l|}{ Previous births } \\
\hline First child & $-1.43(-7.266,4.405)$ & 0.629 \\
\hline Second child & $0.384(-3.873,4.641)$ & 0.858 \\
\hline Third or more & Reference & \\
\hline \multicolumn{3}{|l|}{ Clinical conditions } \\
\hline Gingival Index & $-6.829(-13.869,0.211)$ & 0.057 \\
\hline No.of decayed teeth & $-0.9(-3.358,1.557)$ & 0.470 \\
\hline \multicolumn{3}{|l|}{ Intervention } \\
\hline Papacarie & $4.03(0.652,7.409)$ & $0.020^{*}$ \\
\hline ART & Reference & \\
\hline
\end{tabular}

Adjusted $\mathrm{R}^{2}=0.112, \mathrm{~F}=3.25, \mathrm{P}=0.001$

Cl: confidence interval

*statistically significant at $p \leq 0.05$

\section{Discussion}

Minimally invasive caries removal using Papacarie-Duo and ART significantly improved OHRQoL of pregnant women after one and six months. After adjusting for confounders, Papacarie-Duo group showed greater reduction in OHIP-14 scores, therefore, the null hypothesis can be rejected. 
These findings have implications for the dental care of pregnant women with low or moderate socioeconomic status who suffer from mild to moderate dental pain due to caries combined with possible misconception about dental treatment and limited access to regular dental care. These factors put them at risk for negative impact on their health and well-being. Keeping good oral health helps ensure safe pregnancy and good quality of life[11]. Such treatment modalities may, thus, reduce inequalities in access to care between pregnant women from different socio-economic levels and ensure least negative impact on their quality of life.

This study is probably the first to compare the effect of two minimally invasive caries removal methods on OHRQoL of pregnant women. There is only one previous study conducted in Brazil[37] that compared the effect of minimally invasive caries removal methods (Carisolv and ART) on the longevity of restorations among pregnant women with no assessment of their effect on OHRQoL. The study reported that both methods were successful which is consistent with the present findings despite differences in materials used and length of the follow up periods.

There is a scarcity of studies assessing the impact of minimally invasive restorative techniques on the OHRQoL in pregnant women and in adults in general. Thus, direct comparison with previous studies is not possible. However, the current results are in agreement with a systematic review reporting significant improvement in OHRQoL of different population groups after caries treatment [38] and with Paula et al[39] who reported significant reduction in the overall and domain scores of OHRQoL after treatment with ART among children.

The mean OHIP-14 scores at baseline of the present study were substantially greater than that of pregnant women in India[35], Brazil[40] and China[41], and relatively higher than a study conducted in another area of southeastern Brazil[11]. This suggests possibly greater impact of dental pain and dental problems on the quality of life of women in the present study and may be explained by greater duration or amount of unmet dental treatment needs or greater awareness of women of the impact of these problems on their quality of life. Further research is; therefore, needed to assess factors affecting this impact on OHRQoL and why it is more negative than that of women in countries having similar income levels.

Success rate of restorations were in line with that of other studies which reported success rates to be $100 \%$ among pregnant women in the chemo-mechanical caries removal agents group and $97.6 \%$ for the ART group after 12 months[37]. Relatively less success rate in the present study may be attributed to the loss to follow up and assigning the worst score to lost cases which were considered a failure following the intention-to-treat analysis. Restoration success; meanwhile, had a significant impact on OHRQoL improvement, as noted by the significant greater reduction in OHIP-14 scores after 6 months in patients where restorations were successful. This indicates that the impact on OHRQoL is mainly related to the effectiveness of the restoration and not just an effect attributed to receiving the treatment regardless of its success. 
The present study revealed that besides being effective in caries treatment, Papacarie-Duo had greater impact on OHRQoL of expectant mothers with mild to moderate dental pain. It is important to include patient-reported outcome measures (PROMs) in clinical trials besides clinical and dentist-assessed measures. OHRQoL captures the impact of treatment modalities from patients' perspectives and this is critical for interventions addressing oral health problems that are partly perpetuated by patient behavior or misconception such as in the present study.

One of the limitations of this study is the tendency to report lower impact of oral health because of social desirability bias. In addition, patients with poor oral health and poor expectation may not consider themselves to have poor OHRQoL and consequently report lower scores on OHIP-14. Future studies with longer follow-up periods to assess the long-term effect of minimal invasive caries removal methods are, hence, needed.

It is recommended to integrate chemo-mechanical caries removal method by Papacarie-Duo, which does not need extensive training of dental health care professionals nor sophisticated equipment, as a part of the WHO proposed Basic Package of Oral Care[42] which includes pain relief, preventive, promotional and atraumatic restorative treatments to pregnant women in primary health centers in low-and middleincome countries. Integrating this package with their routine antenatal health care program, would be of great advantage and need to be addressed in future research in order to encourage health promotion required for the control and prevention of dental caries.

\section{Conclusion}

Significant improvement in OHRQoL of pregnant women presented with mild and moderate dental caries occurred after the use of Papacarie-Duo and ART. Providing dental care to pregnant women from modest socio-economic backgrounds in low-resource settings helps ensure their well-being and improve their quality of life which reflects on their general health. Including patient-reported outcomes measures like OHRQoL enhances our understanding of the relationship between oral health and patient's well-being which can be used to inform public policy authorities and help eradicate oral health disparities.

\section{Abbreviations}

ART: Atraumatic Restorative Treatment; ICDAS: International Caries Detection and Assessment System; GI: Gingival Index; OHRQoL: Oral Health Related Quality of Life; GIC: Glass lonomer Cement; SPSS: Statistical package for social sciences; OHIP-14: Oral Health Impact Profile-14; PROM: Patient-Reported Outcome Measures.

\section{Declarations}

Acknowledgements 
The authors would like to express deep gratitude and appreciation to Prof.Maha El Tantawi for her valuable and constructive suggestions during the planning and development of this research work.

\section{Funding}

The research did not receive any fund from public, commercial or not-for-profit sectors.

\section{Availability of data and materials}

The dataset used in this research is available at synapse.org under the title: Impact of minimally invasive restorative techniques on pregnant women oral health related quality of life. Synapse ID: syn23538614. Username: @may.adham

\section{Authors' contributions}

MA conceptualized the study, collected data, applied the intervention, developed and implemented the statistical analysis and wrote the manuscript draft. ME, WA and AR helped designing the study and contributed to the review and editing of the manuscript. All authors read and approved the final manuscript.

\section{Competing interests}

Authors declare that they have no competing interests

\section{Consent for publication}

Not applicable

\section{Ethics approval and consent to participate}

This study was approved by the Research Ethics Committee, Faculty of Dentistry, Alexandria University (IRB 00010556 - IORG 0008839) and was registered under the protocol ID NCT04619264 (https://clinicaltrials.gov/) according to the CONSORT statement of the updated guidelines for reporting randomized clinical trials. Each participant was provided with a written informed consent form. All the information including personal details and clinical information that have been used for this study were de-identified.

\section{References}

1. Petersen PE, Bourgeois D, Ogawa H, Estupinan-Day S, Ndiaye C. The global burden of oral diseases and risks to oral health. Bull World Health Organ. 2005;83:661-9.

2. Mobarak EH, Shabayek MM, Mulder J, Reda AH, Frencken JE. Caries experience of Egyptian adolescents: does the atraumatic restorative treatment approach offer a solution? Med Princ Pract Int J Kuwait Univ Health Sci Cent. 2011;20:545-9. 
3. Kikwilu EN, Frencken J, Mulder J. Impact of Atraumatic Restorative Treatment (ART) on the treatment profile in pilot government dental clinics in Tanzania. BMC Oral Health. 2009;9:14.

4. Helderman $\mathrm{W}$ van P, Benzian H. Implementation of a Basic Package of Oral Care: towards a reorientation of dental Ngos and their volunteers. Int Dent J. 2006;56:44-8.

5. Matsumoto SFB, Motta LJ, Alfaya TA, Guedes CC, Fernandes KPS, Bussadori SK. Assessment of chemomechanical removal of carious lesions using Papacarie Duo ${ }^{\mathrm{TM}}$ : Randomized longitudinal clinical trial. Indian J Dent Res. 2013;24:488.

6. Gajendra S, Kumar JV. Oral health and pregnancy: a review. N Y State Dent J. 2004;70:40-4.

7. Russell SL, Mayberry LJ. Pregnancy and oral health: a review and recommendations to reduce gaps in practice and research. MCN Am J Matern Child Nurs. 2008;33:32-7.

8. Vt H, Manigandan T, Sarumathi T, V A, A A. Dental Considerations in Pregnancy-A Critical Review on the Oral Care. J Clin Diagn Res JCDR. 2013;7:948-53.

9. Krüger M, Lang C, Almeida L, Correa F, Romano A, Pappen F. Dental Pain and Associated Factors Among Pregnant Women: An Observational Study. Matern Child Health J. 2014;19.

10. Mckee MD, Cunningham M, Jankowski KR, Zayas L. Health-related functional status in pregnancy: relationship to depression and social support in a multi-ethnic population. Obstet Gynecol. 2001;97:988-93.

11. Musskopf ML, Milanesi FC, Rocha JM da, Fiorini T, Moreira CHC, Susin C, et al. Oral health related quality of life among pregnant women: a randomized controlled trial. Braz Oral Res [Internet]. Sociedade Brasileira de Pesquisa Odontológica; 2018 [cited 2020 May 30];32. Available from: http://www.scielo.br/scielo.php?script=sci_abstract\&pid=S1806$83242018000100201 \& \operatorname{lng}=$ en\&nrm=iso\&tlng=en

12. Haas JS, Jackson RA, Fuentes-Afflick E, Stewart AL, Dean ML, Brawarsky P, et al. Changes in the health status of women during and after pregnancy. J Gen Intern Med. 2005;20:45-51.

13. J Kittu, V Aditi, Pramila M. Measuring the Impact of Oral Health Related Quality of Life from Womb till Post-Partum. Acta Scientific Dental Sciences. 2019;3:75-82.

14. Rockville, Maryland: US Department of Health and Human Services, National Institute of Dental and Craniofacial Research, National Institute of Health; 2000. DHHS Oral health in America: A report of the Surgeon General; p. 7.

15. Bennadi D, Reddy CVK. Oral health related quality of life. J Int Soc Prev Community Dent. 2013;3:16.

16. Bettie NF, Ramachandiran H, Anand V, Sathiamurthy A, Sekaran P. Tools for evaluating oral health and quality of life. J Pharm Bioallied Sci. 2015;7:S414-9.

17. Slade GD, Spencer AJ. Development and evaluation of the Oral Health Impact Profile. Community Dent Health. 1994;11:3-11.

18. Retori P do C, Knorst JK, Bolsson GB, Savian CM, Casarin M, Santos BZ. Associação entre a higiene bucal e qualidade de vida relacionada à saúde bucal de gestantes. Res Soc Dev. 
2020;9:e137911811-e137911811.

19. Gugnani N, Pandit I, Srivastava N, Gupta M, Sharma M. International Caries Detection and Assessment System (ICDAS): A New Concept. Int J Clin Pediatr Dent. 2011;4:93-100.

20. Wewers ME, Lowe NK. A critical review of visual analogue scales in the measurement of clinical phenomena. Res Nurs Health. 1990;13:227-36.

21. Nadanovsky P, Cohen Carneiro F, Souza de Mello F. Removal of caries using only hand instruments: a comparison of mechanical and chemo-mechanical methods. Caries Res. 2001;35:384-9.

22. Urbaniak, G. C., \& Plous, S. (2013). Research Randomizer (Version 4.0) [Computer software]. Retrieved on June 22, 2013, from http://www.randomizer.org/.

23. Singh S, Singh D, Jaidka S. Comparative clinical evaluation of chemomechanical caries removal agent Papacarie with conventional method among rural population in India - in vivo study. Braz $\mathrm{J}$ Oral Sci. 2011;10:193-8.

24. Frencken JE, Leal SC, Navarro MF. Twenty-five-year atraumatic restorative treatment (ART) approach: a comprehensive overview. Clin Oral Investig. 2012;16:1337-46.

25. Freitas MCC de A, Fagundes TC, Modena KC da S, Cardia GS, Navarro MF de L. Randomized clinical trial of encapsulated and hand-mixed glass-ionomer ART restorations: one-year follow-up. J Appl Oral Sci [Internet]. 2018 [cited 2020 Aug 31];26. Available from:

https://www.ncbi.nlm.nih.gov/pmc/articles/PMC5777411/

26. Al Habashneh R, Khader YS, Salameh S. Use of the Arabic version of Oral Health Impact Profile-14 to evaluate the impact of periodontal disease on oral health-related quality of life among Jordanian adults. J Oral Sci. 2012;54:113-20.

27. Lo EC, Holmgren CJ. Provision of Atraumatic Restorative Treatment (ART) restorations to Chinese pre-school children-a 30-month evaluation. Int J Paediatr Dent. 2001;11:3-10.

28. Motta LJ, Bussadori SK, Campanelli AP, da Silva AL, Alfaya TA, de Godoy CHL, et al. Efficacy of Papacarie ${ }^{\circledR}$ in reduction of residual bacteria in deciduous teeth: a randomized, controlled clinical trial. Clinics. 2014;69:319-22.

29. Khoshnevisan M, Albujeer A, Attaran N, Almahafdha A, Alaboudy A. WHO's oral health assessment questionnaire for adult: psychometric properties of the Arabic version. J Contemp Med Sci. 2016;2.

30. WHO | Oral health surveys: basic methods - 5th edition [Internet]. WHO. World Health Organization; [cited 2020 May 30]. Available from:

http://www.who.int/oral_health/publications/9789241548649/en/

31. Silness J, Loe H. PERIODONTAL DISEASE IN PREGNANCY. II. CORRELATION BETWEEN ORAL HYGIENE AND PERIODONTAL CONDTION. Acta Odontol Scand. 1964;22:121-35.

32. Loe H, Silness J. PERIODONTAL DISEASE IN PREGNANCY. I. PREVALENCE AND SEVERITY. Acta Odontol Scand. 1963;21:533-51.

33. Lawrence HP, Thomson WM, Broadbent JM, Poulton R. Oral health-related quality of life in a birth cohort of 32-year olds. Community Dent Oral Epidemiol. 2008;36:305-16. 
34. Wandera MN, Engebretsen IM, Rwenyonyi CM, Tumwine J, Astrøm AN, PROMISE-EBF Study Group. Periodontal status, tooth loss and self-reported periodontal problems effects on oral impacts on daily performances, OIDP, in pregnant women in Uganda: a cross-sectional study. Health Qual Life Outcomes. 2009;7:89.

35. Acharya S, Bhat PV, Acharya S. Factors affecting oral health-related quality of life among pregnant women. Int J Dent Hyg. 2009;7:102-7.

36. Cornejo C, Rossi G, Rama A, Gomez-Gutierrez N, Alvaredo G, Squassi A, et al. Oral health status and oral health-related quality of life in pregnant women from socially deprived populations. Acta Odontol Latinoam AOL. 2013;26:68-74.

37. Barata TJE, Bresciani E, Mattos MCR, Lauris JRP, Ericson D, Navarro MF de L. Comparison of two minimally invasive methods on the longevity of glass ionomer cement restorations: short-term results of a pilot study. J Appl Oral Sci Rev FOB. 2008;16:155-60.

38. Aimée NR, Damé-Teixeira N, Alves LS, Borges GÁ, Foster Page L, Mestrinho HD, et al. Responsiveness of Oral Health-Related Quality of Life Questionnaires to Dental Caries Interventions: Systematic Review and Meta-Analysis. Caries Res. Karger Publishers; 2019;53:585-98.

39. Paula JS, Tôrres LHN, Ambrosano GMB, Mialhe FL. Association between oral health-related quality of life and atraumatic restorative treatment in school children: An exploratory study. Indian J Dent Res. 2012;23:738.

40. Oliveira BH, Nadanovsky P. Psychometric properties of the Brazilian version of the Oral Health Impact Profile-short form. Community Dent Oral Epidemiol. 2005;33:307-14.

41. Lu H-X, Xu W, Wong MCM, Wei T-Y, Feng X-P. Impact of periodontal conditions on the quality of life of pregnant women: a cross-sectional study. Health Qual Life Outcomes [Internet]. 2015 [cited 2020 Sep 30];13. Available from: https://www.ncbi.nlm.nih.gov/pmc/articles/PMC4446953/

42. Batra, Manu \& Rajwar, Yogesh \& Agarwal, Nimish \& Singh, Arjun \& Dutt, Manas \& Sinha, Abhishek. (2014). BASIC PACKAGE FOR ORAL CARE: A STEP TOWARDS PRIMARY ORAL HEALTH CARE. TMU J Dent. 1. 57-60.

\section{Figures}




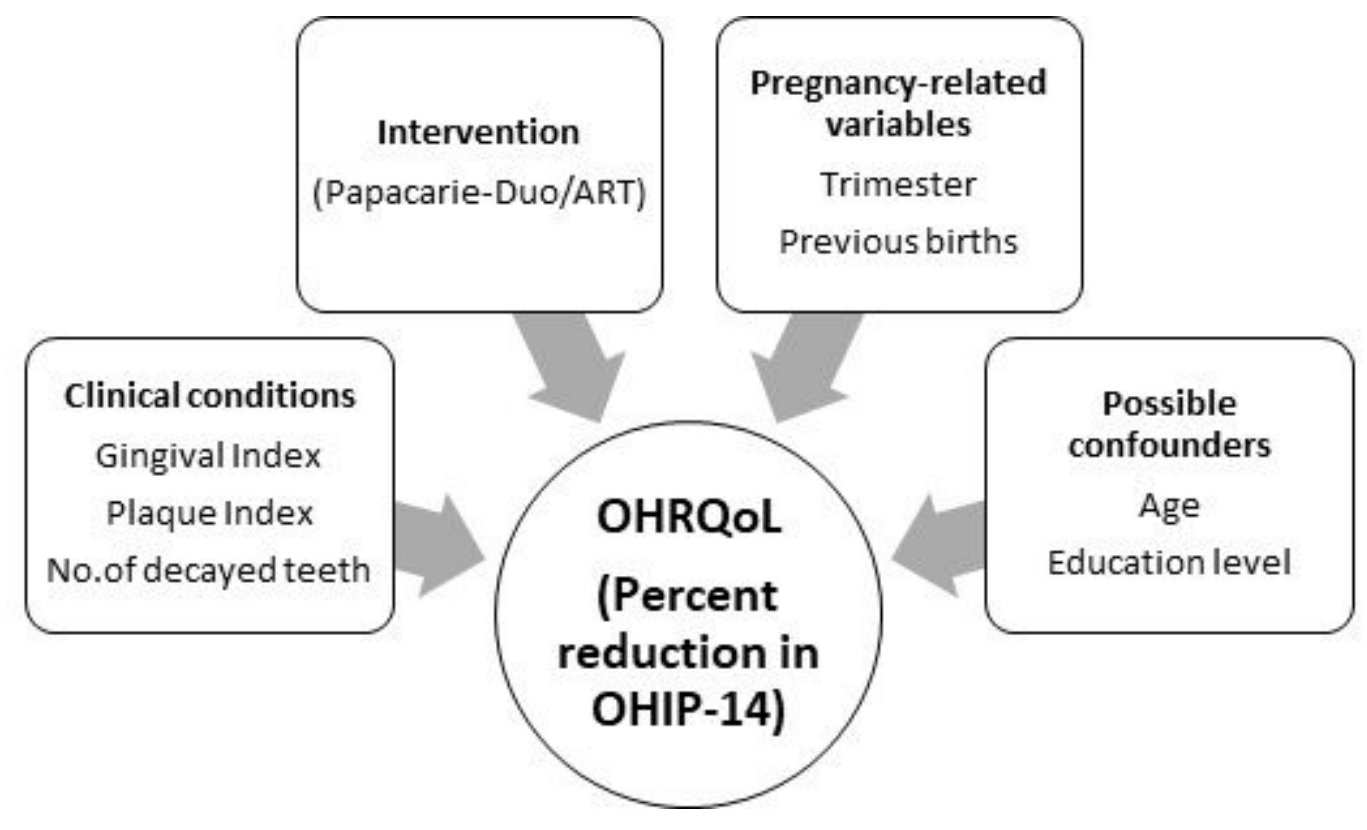

Figure 1

Theoretical framework of the relationships between independent variables and OHRQoL of pregnant women 


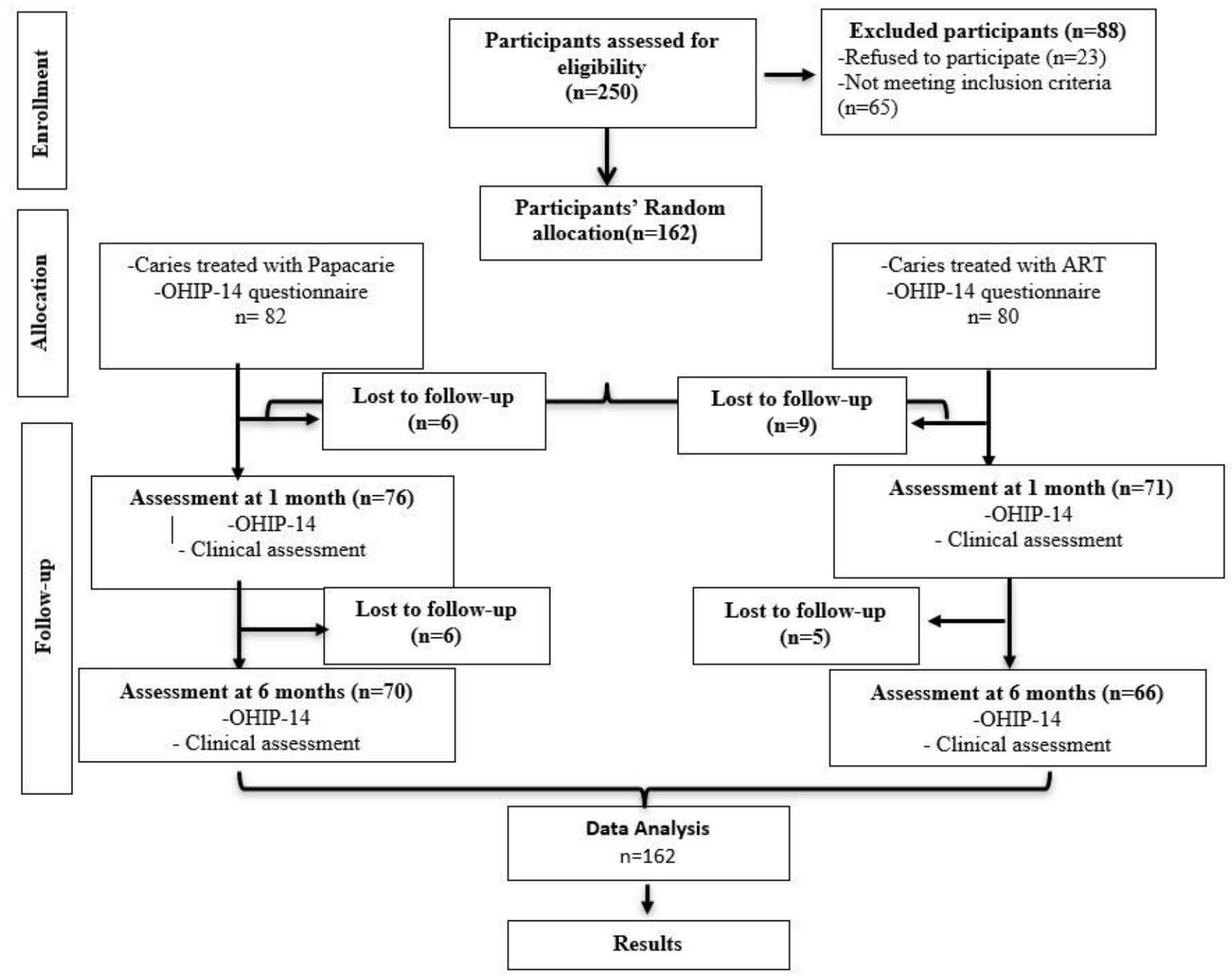

Figure 2

Flow chart

\section{Supplementary Files}

This is a list of supplementary files associated with this preprint. Click to download.

- CONSORT2010ChecklistQoLmanuscript.doc 